\title{
Evaluation of Factors Affecting Perinatal Mortality in Patients with HELLP Syndrome
}

\author{
Reyhan GUNDUZ¹, Senem YAMAN TUNC'1, Mehmet Sait ICEN¹, Sabahattin ERTUGRUL², Talip GUL¹ \\ Diyarbakir, Turkey
}

\section{ABSTRACT}

OBJECTIVE: To determine the perinatal mortality rate in patients with HELLP syndrome in our clinic and to investigate the factors affecting perinatal mortality. It also makes recommendations to reduce perinatal mortality and contributes to the literature.

STUDY DESIGN: Three-hundred-and-eighty-three patients were retrospectively evaluated in this cohort study. The patients' demographic, clinical data, laboratory results, gestational week at delivery, method of delivery, neonatal birth weight, fetal gender, 1- and 5-minute APGAR scores, place of delivery, maternal morbidity, mortality rates, and perinatal mortality rates were recorded. The relationship of these factors with perinatal mortality was investigated.

RESULTS: The rate of perinatal mortality was determined as $6 \%$. Patients with HELLP syndrome who experienced perinatal mortality showed significantly lower birth weight, gestational age at delivery, and 1 - and 5-minute APGAR score values $(p<0.05)$. With respect to methods of delivery, we determined that vaginal delivery was linked to a significantly higher rate of perinatal mortality $(p<0.001)$. Gestational age at delivery, birth weight, 1- and 5-minute APGAR scores were negatively correlated with perinatal mortality. Logistic regression revealed the APGAR score at 5 minutes as the most reliable independent predictive finding for perinatal mortality.

CONCLUSION: We think that to decrease perinatal mortality rates, maternal and fetal well-being in patients with HELLP syndrome should be closely monitored and delivery and follow-up should take place at tertiary health institutions after maternal and neonatal intensive care arrangements are made. Particularly, neonates with low 5-minute APGAR scores in the postpartum evaluation of neonatal condition are recommended to be followed-up at the neonatal intensive care unit.

Keywords: APGAR score, HELLP syndrome, Perinatal mortality, Prematurity

Gynecol Obstet Reprod Med 2021;27(3):213-220

\footnotetext{
Dicle University, Faculty of Medicine, Department of Obstetrics and Gynecology. Diyarbakir, Turkey

${ }^{2}$ Dicle University, Faculty of Medicine Department of Pediatrics, Diyarbakir, Turkey

Address of Correspondence: Reyhan Gunduz

University of Dicle, School of Medicine,

Department of Obstetrics and Gynecology

Diyarbakir, Turkey

ryhn.gunduz@gmail.com
}

Submitted for Publication: 24.08.2020 Revised for Publication: 04.02.2021 Accepted for Publication: 23.03.2021 Online Published: 31.03.2021

ORCID IDs of the authors: $\quad$ RG: 0000-0001-8468-7038

SYT: 0000-0003-2583-277 MSI: 0000-0001-7982-1407

SE: 0000-0003-0903-6520 TG: 0000-0002-6941-6851

\begin{tabular}{c|c}
\hline Quick Response Code: & Access this article online \\
\cline { 2 - 2 } & Website: www.gorm.com.tr \\
\cline { 2 - 2 } & e- mail: info@gorm.com.tr \\
\hline
\end{tabular}

How to cite this article: Gunduz R. Yaman Tunc S. Icen MS. Ertugrul S. Gul T. Evaluation of Factors Affecting Perinatal Mortality in Patients with HELLP Syndrome. Gynecol Obstet Reprod Med. 2021;27(3):213-220

\section{Introduction}

HELLP syndrome was first defined in 1982 by Weinstein and the term "HELLP" is an acronym that describes a syndrome that is used to define a clinical condition causing hemolysis, elevated liver enzyme levels, and a low platelet count (1). Certain authors consider HELLP syndrome to be a disease distinct from preeclampsia as $15-20 \%$ of patients who have HELLP syndrome do not manifest hypertension or proteinuria (2).

Generally, HELLP syndrome is encountered in 0.5 to $0.9 \%$ of pregnant women (1). Approximately 1 to $2 \%$ of women with severe preeclampsia/eclampsia have microangiopathic hemolysis, which can be evaluated as HELLP syndrome.

History of previous preeclampsia or HELLP syndrome constitutes a risk factor for HELLP syndrome. Various genetic variants have been reported in relation to an increased risk for HELLP syndrome, however, these do not play any role in clinical management (3). Different from preeclampsia, nulliparity does not constitute a risk factor for HELLP syndrome (4). 
Multiparous patients comprise no less than half of the affected patients.

HELLP syndrome has a variable presentation (5). Generally, symptoms have a rapid onset and they progressively deteriorate. On physical examination, approximately $85 \%$ of patients show hypertension (defined as blood pressure $\geq 140 / 90 \mathrm{mmHg}$ ) and proteinuria; however, it is important to express that one or both of these may not be detected in women with severe HELLP syndrome (5).

Symptoms usually develop between 27 and 37 weeks' gestation and $30 \%$ of the cases become manifest postpartum (1). In general, the rate of perinatal mortality varies between $7 \%$ and $20 \%$ and its leading causes include preterm delivery, intrauterine growth retardation, and placental detachment complications $(5,6)$. Preterm delivery is common, with a rate of $70 \%$ (15\% occur before 27 weeks' gestation) (7).

This study aims to determine the rate of perinatal mortality in patients diagnosed with HELLP syndrome in the antepartum or postpartum period who presented to our tertiary hospital, which serves as a reference hospital in the Southeastern Anatolia Region, for 10 years, to make suggestions towards decreasing this rate by identifying the factors that influence mortality, and hence, to contribute to the literature.

\section{Materials and method}

This retrospective cohort study included 383 patients who had been admitted to the Gynecology and Obstetrics Clinic of Dicle University, Faculty of Medicine for a diagnosis of HELLP in the antepartum or postpartum period between January 2010-January 2020. The study was reviewed and approved by the ethics committee of Dicle University Faculty of Medicine (Ethics approval reference number: 140, date: 07.05.2020). All procedures were performed according to the Declaration of Helsinki.

Exclusion criteria were as follows: risk factors other than HELLP syndrome that could influence perinatal mortality such as a gestational age $<20$ weeks, chronic diseases, acute fatty liver of pregnancy, presence of thrombotic thrombocytopenic purpura or hemolytic-uremic syndrome, smoking or alcohol intake during pregnancy, abnormal placental invasion, placental abruption, multiple pregnancies; and failure to meet the diagnostic criteria for HELLP syndrome. Patient data including age, gravidity, parity, method of delivery, gestational age at delivery, place of delivery, laboratory results, neonatal birth weight, fetal gender, 1- and 5-minute APGAR scores, systolic/diastolic blood pressure, maternal mortality, and maternal complications, and data regarding postpartum corticosteroid use were obtained by reviewing the hospital information management system archives and patient files.

The diagnosis of HELLP syndrome was made in accor- dance with the diagnostic criteria recommended by The American College of Obstetricians and Gynecologists (ACOG) (8). Cases we considered as perinatal mortality were determined based on the definitions used by the World Health Organization (WHO) and the National Center for Health Statistics (NCHS) (9). According to this definition; neonatal deaths that occurred within 7 days of delivery and fetal deaths that occurred at 28 weeks' gestation or later were accepted as perinatal mortality. Gestational age was determined based on the date of the last menstrual period or by ultrasound measurement for patients who did not know the date of their last menstrual period. Birth weights, 1- and 5-minute APGAR scores were determined by the neonatologists attending the deliveries. Scores were expressed quantitatively. As laboratory results, values at initial admission were recorded. Patients who met the diagnostic criteria for HELLP during pregnancy were accepted as antepartum HELLP syndrome cases and those who met the diagnostic criteria after labor was accepted as postpartum HELLP syndrome cases. With respect to the place of delivery, patients who delivered at our hospital and patients who were referred to our hospital after undergoing labor at other health institutions were categorized into the tertiary hospital group and the external center group, respectively.

Patients diagnosed with HELLP syndrome at external centers, for whom a referral request was sent to our hospital, were accepted after the arrangement of neonatal and maternal intensive care unit conditions. These conditions were also primarily ensured for our hospitalized patients. Patients diagnosed with HELLP syndrome at our clinic were admitted to the intensive care unit at our clinic. At our clinic; patients at more than 34 weeks' gestation and patients with intrauterine death were routinely planned to undergo delivery immediately after maternal stabilization. In the absence of these two findings, magnesium sulfate therapy was administered (a loading dose of $6 \mathrm{~g}$ over $20 \mathrm{~min}$, followed by a maintenance dose of 2 $\mathrm{g} / \mathrm{h}$ as a continuous intravenous solution for at least $24 \mathrm{~h}$ postpartum) to patients between 24-32 weeks of gestation to prevent convulsions and for fetal/neonatal neuroprotection. Corticosteroids (12 mg betamethasone intramuscularly every $12 \mathrm{~h}$, twice) for fetal lung maturation were given to fetuses $\leq 34$ weeks' gestation $(5,10)$. With respect to delivery method; vaginal delivery was preferred primarily in patients without contraindications for vaginal delivery who showed favorable Bishop scores. On the other hand, cesarean delivery was preferred primarily for pregnancies at less than 32 weeks of gestation with oligohydramnios and growth retardation (5).

Statistical analyses were conducted using the SPSS 21 statistics software package. Descriptive data were presented as mean, standard deviation, median, minimum, maximum, frequency, and percentages. Data of patients who showed perinatal mortality and those who did not were compared and the Kolmogorov-Smirnov test was used to assess data for normality. Categorical data were analyzed using the Chi-square test. 
Normally distributed continuous variables were compared using Student's t-test, and pairwise comparisons of continuous variables without a normal distribution used the non-parametric Mann Whitney U test. Spearman correlation and logistic regression analysis were performed to determine the factors that influenced perinatal mortality. A p-value $<0.05$ was considered statistically significant.

\section{Results}

Between the years 2010 and 2020, the total number of deliveries recorded at our clinic was 16.548, 4.473 (27\%) patients diagnosed with preeclampsia and 242 (1.4\%) patients diagnosed with eclampsia. Three hundred eighty-three (2.3\%) patients were determined to have been diagnosed with antepartum or postpartum HELLP syndrome during the 10-year period. Of the 383 patients who underwent labor with a diagnosis of HELLP diagnosis, 23 (6\%) were found to have experienced perinatal mortality.

Mean patient age was $30.2 \pm 6.9$, mean gestational week at delivery was $31.9 \pm 4.2$, mean neonatal birth weight was 1779.4 \pm 776.08 , mean 1-minute APGAR score was 4.7 \pm 2.1 , and mean 5-minute APGAR score was 6.7 \pm 2.2 . Demographic, clinical, and laboratory data are shown in table I.

Table I: Evaluation of demographic, clinical, and laboratory findings in the patients with HELLP Syndrome

\begin{tabular}{ll}
\hline & Mean \pm SD, (Min-Max) \\
\hline Age & $30.2 \pm 6.9,(14-49)$ \\
Gravidity & $3.8 \pm 3.3,(1-34)$ \\
Parity & $2.4 \pm 2.5,(0-11)$ \\
Gestational week at delivery & $31.9 \pm 4.2,(20-40)$ \\
Neonatal birth weight (gr) & $1779.4 \pm 776.08,(240-4100)$ \\
1-minute APGAR scores & $4.7 \pm 2.1,(0-10)$ \\
5-minute APGAR scores & $6.7 \pm 2.2,(0-10)$ \\
Hematocrit (\%) & $36.1 \pm 5.65,(15-49)$ \\
Hemoglobin (g/dl) & $12.2 \pm 2.8,(5-45)$ \\
Platelet (K/uL) & $103.3 \pm 64.6,(12.2-735.5)$ \\
ALT (U/L) & $159.5 \pm 171.2,(7-1526)$ \\
AST (U/L) & $245.9 \pm 277.9,(16-1572)$ \\
LDH (IU/L) & $864.6 \pm 589.8,(114-4840)$ \\
Total Bilirubin (mg/dl) & $3.1 \pm 25.6,(0-457)$ \\
Urea (mg/dl) & $28.9 \pm 16.3,(0.6-138)$ \\
Creatinine (mg/dl) & $1.2 \pm 6.0,(0.2-93)$ \\
Proteinuria & $1.6 \pm 0.82,(0-3)$ \\
\hline
\end{tabular}

The mean age of 23 patients with perinatal mortality was $33.3 \pm 6.07$, mean gestational week at delivery was $25.8 \pm 3.8$, the median value for the gestational week at delivery was 25 (20-33) weeks, mean neonatal birth weight was $888.4 \pm 472.6$, mean 1-minute APGAR score was $0.4 \pm 1.2$ and mean 5-minute APGAR score was $0.5 \pm 1.6$ (Table II).
Demographic, laboratory, and clinical data were compared between the group that showed perinatal mortality and the group that did not. Birth weight, gestational age at delivery, and 1- and 5-minute APGAR scores were statistically lower in the perinatal mortality group $(p<0.05)$ (Table II). Other data shown in table II did not demonstrate any difference between these groups.

No statistically significant difference was found in the age and neonatal birth weight between patients with and without perinatal mortality based on the average birth weeks of patients with perinatal mortality $(p>0.05)$. 1 - and 5-minute APGAR scores and the gestational week at delivery were significantly lower in the group with perinatal mortality $(p<0.05)$.

Of the 383 patients included in our study, the pregnancy was concluded with vaginal delivery in $67(17.5 \%)$ and with cesarean delivery in $316(82.5 \%)$. The perinatal mortality rate was significantly higher in patients who underwent vaginal delivery $(p<0.001)$. Maternal laboratory results, whether HELLP syndrome was postpartum or antepartum, fetal gender, the health institution at which delivery occurred, presence or absence of postpartum corticosteroid use, maternal morbidity, maternal monitoring in the intensive care unit, and maternal mortality were determined to not affect perinatal mortality $(p>0.05)$ (Table III).

Median (minimum-maximum) values of demographic data (for patients with perinatal mortality) for vaginal deliveries at average birth weeks were calculated. Gravidity was 4 (1-9), parity was 3 (0-9), a gestational week at delivery was 25 (2032) weeks, 1-minute APGAR scores were 0 (0-5), 5-minute APGAR scores were 0 (0-7). There was no significant difference between the patients who had a vaginal delivery with and without perinatal mortality in terms of the gestational week at delivery $(p>0.05)$.

The number of patients with postpartum HELLP syndrome was 108 (28.2\%). When we compared our patients with postpartum and antepartum HELLP syndrome in terms of age, neonatal birth weight, gravidity, parity, a gestational week at delivery, 1-minute and 5-minute APGAR score, parity was significantly different between the two groups $(p<0.05)$ (Table IV).

Correlational analysis of factors influencing perinatal mortality revealed that perinatal mortality had a moderate negative correlation with 1-minute and 5-minute APGAR scores, and a weak correlation with gestational age at delivery and birth weight (Table V). Logistic regression analysis showed that a low 5-minute APGAR score caused a 13.9-fold increase in perinatal mortality, identifying it as the most reliable independent predictive value (Table VI). 
Table II: Comparison of factors affecting perinatal mortality between groups

\begin{tabular}{|c|c|c|c|}
\hline \multirow[b]{2}{*}{$n=383$} & \multicolumn{2}{|c|}{ Perinatal mortality } & \multirow[b]{2}{*}{$p$} \\
\hline & 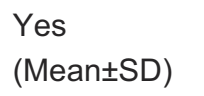 & $\begin{array}{l}\text { No } \\
\text { (Mean } \pm S D)\end{array}$ & \\
\hline Age & $33.3 \pm 6.07$ & $30.03 \pm 6.97$ & 0.153 \\
\hline Neonatal birth weight (g) & $888.4 \pm 472.6$ & $1839.7 \pm 755.8$ & 0.019 \\
\hline Gravidity & $4.4 \pm 3.5$ & $3.8 \pm 3.3$ & 0.553 \\
\hline Parity & $2.8 \pm 2.8$ & $2.4 \pm 2.4$ & 0.607 \\
\hline Gestational week at delivery & $25.8 \pm 3.8$ & $32.4 \pm 3.8$ & $<0.001$ \\
\hline 1-minute APGAR scores & $0.4 \pm 1.2$ & $4.9 \pm 1.8$ & $<0.001$ \\
\hline 5-minute APGAR scores & $0.5 \pm 1.6$ & $7.1 \pm 1.5$ & $<0.001$ \\
\hline Hematocrit $(\%)$ & $36.0 \pm 6.2$ & $36.1 \pm 5.5$ & 0.181 \\
\hline Hemoglobin (g/dL) & $12.2 \pm 2.1$ & $12.1 \pm 2.3$ & 0.633 \\
\hline Platelet (K/uL) & $76 \pm 38.5$ & $105 \pm 65.8$ & 0.024 \\
\hline Alanine aminotransferase (U/L) & $193.6 \pm 322.0$ & $157.4 \pm 158.4$ & 0.751 \\
\hline Lactate dehydrogenase (IU/L) & $957.1 \pm 580.1$ & $861 \pm 590.9$ & 0.264 \\
\hline Aspartate aminotransferase (U/L) & $270.3 \pm 365.7$ & $246.04 \pm 274.3$ & 0.684 \\
\hline Total bilirubin (mg/dL) & $1.4 \pm 1.84$ & $3.3 \pm 27.03$ & 0.379 \\
\hline Urea (mg/dL) & $31.7 \pm 14.8$ & $28.4 \pm 15.5$ & 0.149 \\
\hline Creatinine (mg/dL) & $0.7 \pm 0.2$ & $1.3 \pm 6.2$ & 0.500 \\
\hline Proteinuria & $1.5 \pm 0.9$ & $1.6 \pm 0.8$ & 0.655 \\
\hline Maternal intensive care unit period (days) & $1.7 \pm 4.3$ & $0.9 \pm 3.2$ & 0.155 \\
\hline Systolic blood pressure $(\mathrm{mmHg})$ & $156.3 \pm 31.2$ & $155.7 \pm 20.5$ & 0.550 \\
\hline Diastolic blood pressure (mmHg) & $96.8 \pm 18.3$ & $97.2 \pm 12.6$ & 0.996 \\
\hline
\end{tabular}

Data are presented as mean $\pm S D$, Independent t-test, Mann Whitney U Test, $p<0.05$ statistically significant (in bold)

Table III: Percentage distribution of factors affecting perinatal mortality

\begin{tabular}{|c|c|c|c|c|}
\hline & & $\begin{array}{c}\text { Patients with } \\
\text { HELLP syndrome } \mathrm{n}(\%)\end{array}$ & $\begin{array}{l}\text { Rate of perinatal } \\
\text { mortality } n(\%)\end{array}$ & $p$ \\
\hline \multirow[t]{2}{*}{ Method of delivery } & Vaginal Delivery & $67(17.5)$ & $11(16.4)$ & 0.000 \\
\hline & Caesarean delivery & $316(82.5)$ & $12(3.79)$ & \\
\hline \multirow[t]{2}{*}{ Thrombocytopenia } & Yes & $327(86.3)$ & $22(6.72)$ & 0.173 \\
\hline & No & $56(13.7)$ & $1(1.78)$ & \\
\hline \multirow[t]{2}{*}{ Anemia } & Yes & $60(15.7)$ & $5(8.3)$ & 0.345 \\
\hline & No & $323(84.3)$ & $18(5.57)$ & \\
\hline \multirow[t]{2}{*}{ Postpartum HELLP } & Yes & $108(28.2)$ & $6(5.55)$ & 0.817 \\
\hline & No & $275(71.8)$ & $17(6.18)$ & \\
\hline \multirow[t]{2}{*}{ Place of delivery } & External center & $54(14)$ & $3(5.55)$ & 0.663 \\
\hline & Tertiary hospital & $329(86)$ & $20(6.07)$ & \\
\hline \multirow[t]{2}{*}{ Maternal mortality } & Yes & $3(0.8)$ & $1(33.3)$ & 0.051 \\
\hline & No & $380(99.2)$ & $22(5.7)$ & \\
\hline \multirow[t]{2}{*}{ Maternal intensive care unit } & Yes & $62(16.2)$ & $6(9.6)$ & 0.147 \\
\hline & No & $321(83.8)$ & $17(5.3)$ & \\
\hline \multirow[t]{2}{*}{ Postpartum corticosteroid } & Yes & $262(68.4)$ & $14(5.3)$ & 0.393 \\
\hline & No & $121(31.6)$ & $9(7.4)$ & \\
\hline \multirow[t]{2}{*}{ Maternal complication } & Yes & $28(7.3)$ & $2(7.1)$ & 0.975 \\
\hline & No & $355(92.7)$ & $21(5.9)$ & \\
\hline \multirow[t]{2}{*}{ Fetal gender } & Male & $197(51.4)$ & $9(4.6)$ & 0.378 \\
\hline & Female & $186(48.6)$ & $14(7.5)$ & \\
\hline \multirow[t]{2}{*}{ Parity } & Nulliparous & $137(35.8)$ & $8(5.8)$ & \\
\hline & Multiparous & $246(64.2)$ & $15(6.09)$ & 0.933 \\
\hline
\end{tabular}

Data are presented as percent, Chi-square test, $p<0.05$ statistically significant (in bold) 
Table IV: Comparison of patients with postpartum and antepartum HELLP syndrome

\begin{tabular}{lccc}
\hline & $\begin{array}{c}\text { Postpartum } \\
\text { HELLP } \\
\mathrm{n}=383\end{array}$ & $\begin{array}{c}\text { Antepartum } \\
\text { HELLP } \\
\mathrm{n}=275\end{array}$ & $p$ \\
\cline { 2 - 4 } & $($ Mean $\pm \mathrm{SD})$ & $($ Mean \pm SD) & 0.963 \\
Age & $29.7 \pm 6.9$ & $30.5 \pm 6.9$ & $1780.2 \pm 767.6$ \\
Geonatal birth weight (g) & $1774.9 \pm 836$ & $3.9 \pm 3.4$ & 0.646 \\
Parity & $3.7 \pm 2.9$ & $2.3 \pm 2.5$ & 0.711 \\
Gestational week at delivery & $2.7 \pm 2.3$ & $32.0 \pm 4.0$ & 0.016 \\
1-minute APGAR scores & $31.6 \pm 4.8$ & $4.6 \pm 2.1$ & 0.798 \\
5-minute APGAR scores & $5.0 \pm 2.0$ & $6.6 \pm 2.2$ & 0.239 \\
\hline
\end{tabular}

Data are presented as mean $\pm S D$, Independent $t$-test, Mann Whitney $U$ Test, $p<0.05$ statistically significant (in bold)

Table V: Spearman correlation analysis of factors affecting perinatal mortality

\begin{tabular}{lll}
\hline $\mathrm{n}=383$ & $\boldsymbol{p}$ & $\boldsymbol{r}$ \\
\hline Age & 0.034 & $0.110^{*}$ \\
Gestational week at delivery & 0.000 & $-0.343^{* *}$ \\
Neonatal birth weight (g) & 0.000 & $-0.305^{* *}$ \\
1-minute APGAR scores & 0.000 & $-0.404^{* *}$ \\
5-minute APGAR scores & 0.000 & $-0.419^{* *}$ \\
Platelet & 0.024 & $-0.118^{*}$ \\
\hline
\end{tabular}

Spearman correlation analysis

Table VI: Binary logistic regression analysis of factors affecting perinatal mortality

\begin{tabular}{lllll}
\hline $\mathrm{n}=383$ & BETA & Wald & $p$ & $\% 95 \mathrm{Cl}$ \\
\hline Gestational week at delivery & 0.080 & 0.081 & 0.776 & $0.626 / 1.874$ \\
Neonatal birth weight $(\mathrm{gr})$ & 0.000 & 0.038 & 0.846 & $0.996 / 1.003$ \\
1-minute APGAR scores & 0.512 & 0.826 & 0.363 & $0.553 / 5.027$ \\
5-minute APGAR scores & -1.675 & 13.940 & $<0.001$ & $0.078 / 0.451$ \\
Platelet & -0.010 & 0.451 & 0.502 & $0.962 / 1.019$ \\
\hline
\end{tabular}

Binary logistic regression

\section{Discussion}

Ethnic origin, gestational age, and multiple pregnancies can be counted among the main factors that influence perinatal mortality rates. The perinatal mortality rate varies depending on these factors (11). In the present study, we aimed to investigate the factors that could affect this rate, examine the relationship between these factors and mortality, make suggestions that could decrease perinatal mortality rates, and reduce mortality rates in patients with HELLP syndrome, among whom the rate of perinatal mortality was determined as $6 \%$. As a result of this study, we identified the 5-minute APGAR score as the most valuable predictive finding for perinatal mortality in patients with HELLP syndrome.

The rate of HELLP syndrome was determined as $0.6 \%$ in a study conducted by Guzel et al. (12). Meanwhile, we determined this rate as $2.3 \%$ in the present study. We think that this difference is related to the inclusion of pregnant women diagnosed with HELLP syndrome during both the postpartum and the antepartum period and the high admission rate of patients diagnosed with HELLP syndrome in nearby provinces to our clinic during the years included in this study. Accordingly, our study determined the number of patients with HELLP syndrome in only the antepartum period as $275(1.6 \%)$.

In the literature, the rate of perinatal mortality is reported to be between $7 \%$ and $20 \%(5,6)$. In the present study, this rate was determined as $6 \%$. We reason that our rate was close to the lower range in the literature because our patients with HELLP syndrome underwent labor after neonatal intensive care conditions were arranged. Furthermore, this rate also includes patients who underwent labor at external centers and were admitted in the postpartum period. These patients underwent labor in secondary hospitals. Therefore, we recommend that patients with HELLP syndrome should undergo labor at 
tertiary hospitals after intensive care conditions are arranged and the required equipment is prepared. In a study by Sadaf et al., the perinatal mortality rate was determined as $57.5 \%$ (13). The authors explained this by the low birth weights and the high number of preterm deliveries encountered in patients with HELLP syndrome. Moreover, they also implicated late diagnosis due to the variable clinical presentation of HELLP syndrome and late presentation to health centers.

In a study conducted by Y1ldirim et al., patients with HELLP syndrome presented a mean age of 28 , gestational age of 34 weeks at delivery, birth weight of $940 \mathrm{gr}$, 5-minute APGAR score of 4.8 (14). The results of the present study were in agreement with these values. In both studies, symptoms typically became manifest between 27 and 37 weeks of gestation (1), and in accordance with these gestational weeks, low birth weights and APGAR scores were determined.

Aydin et al. compared patients with complete and partial HELLP syndrome in a study (15). They identified delivery before 34 weeks' gestation as a major complication. There was no difference between the two groups in terms of perinatal mortality. In the present study, we evaluated patients with complete HELLP syndrome, and when we investigated the differences between the groups with and without perinatal mortality, the group with mortality showed significantly lower gestational age at delivery, birth weight, and 1- and 5-minute APGAR score values. We found that the lower were these values, the higher was the rate of mortality. Logistic regression analysis revealed the 5-minute APGAR score as an independent determining predictive value for perinatal mortality in HELLP syndrome. Thus, we think that the earlier is the onset of the diagnosis of HELLP syndrome, the poorer is the perinatal mortality outcome. Therefore, we suggest that a low 5minute APGAR score in the neonatal examination should be considered important and the neonate should be monitored at the neonatal intensive care unit. When we compared the patients based on the average birth weeks in patients with perinatal mortality, we also found that the group with mortality showed significantly lower gestational age at delivery and 1and 5-minute APGAR score values. Based on this result, we think that perinatal mortality may be due to prematurity. More comprehensive studies are needed.

Aydin et al. determined the rate of cesarean delivery as $73.4 \%$ in patients with complete HELLP syndrome (15). In the present study, this rate was found as $82.5 \%$. Upon consideration of the relationship between perinatal mortality and delivery method, vaginal delivery is found to be linked to a significantly higher perinatal mortality rate. It is reported in the literature that the induction of labor at less than 32 weeks of gestation is associated with a high rate of failure even with the use of cervical ripening agents and would likely be prolonged, thus posing a higher complication risk to the mother and the fetus than severe HELLP syndrome (5). We think that the perinatal mortality rate in our study was close to the lower range of the rates reported in the literature due to the use of cesarean delivery in patients at less than 32 weeks' gestation with oligohydramnios and growth retardation, which resulted in high cesarean delivery rates. Therefore, patients at less than 32 weeks' gestation can be primarily recommended cesarean delivery depending on the maternal and fetal well-being states. When we compared the patients with vaginal delivery based on the average birth weeks of patients with perinatal mortality, there was no statistically significant difference between the groups with and without perinatal mortality in the gestational week. This suggests that perinatal mortality will be due to vaginal deliveries during these weeks of gestation rather than prematurity. However, more comprehensive studies are needed.

In contrast to preeclampsia, the literature does not report nulliparity as a risk factor for HELLP syndrome and no less than half of affected patients are multiparous patients (4). In the present study, the rate of multiparous patients was $64.2 \%$ and we determined that being multiparous or nulliparous did not affect perinatal mortality.

The pathogenesis of HELLP syndrome has not been elucidated yet. Upon review of the studies in the literature on the pathogenesis, we found that the authors performed interventions based on the hypothesis that severe preeclampsia/ HELLP syndrome is a systemic inflammatory disorder involving the complement cascade as a key mediator, and on the observational finding that female patients with mutations in complement regulatory proteins show an elevated risk of preeclampsia (16). A meta-analysis conducted in 2015 found that $7 \%$ of 512 patients who had conceived after HELLP syndrome developed HELLP syndrome again in their subsequent pregnancies (17). Therefore, like others, we suggest that more studies should be performed on the pathogenesis of HELLP syndrome. In this way, we should be able to identify these patients before the onset of the adverse maternal and fetal effects of this disease or identify the patients under a high risk by determining the risk factors, and refer these patients to tertiary hospitals. We think that monitoring the pregnancies of these patients at tertiary hospitals can delay the onset of this syndrome in pregnancy, and prevent preterm deliveries, and hence, perinatal mortality.

It has come to our attention that studies on postpartum HELLP syndrome in the literature mostly case reports. We think that this is due to the rarity of postpartum HELLP syndrome or the overlooked diagnosis. Of the few studies on this subject, Sushil et al. In their prospective study, it was stated that there were 24 patients diagnosed with HELLP syndrome, 5 of them $(20 \%)$ were postpartum HELLP syndrome. The total perinatal mortality rate was calculated as $45.8 \%$ in their study. It is not specified whether postpartum HELLP syndrome affects this perinatal mortality rate (18). Our literature review on how postpartum HELLP syndrome influences peri- 
natal mortality revealed that there were not enough studies on this subject. This study did not determine a statistically significant difference between patients with postpartum and antepartum HELLP syndrome in terms of perinatal mortality rates. A study done by Kinay et al. stated that it was not clear whether perinatal morbidity and mortality were linked to the characteristics of the disease or premature delivery (2). We think that this is because the pathogenesis of HELLP syndrome is unclear and the effects of the disease occur during the pregnancy and impact the pregnancy even if the diagnosis or the disease are identified later in the postpartum period. Thus, we think that more studies are needed on this matter.

In a meta-analysis of 11 studies ( $\mathrm{n}=550$ women) on corticosteroid therapy in women with HELLP syndrome, it was found that the administration of steroids did not result in a convincing reduction in maternal mortality or severe maternal morbidity, or perinatal/infant mortality (19). Two studies included in this meta-analysis investigated the effects of betamethasone and dexamethasone, and dexamethasone was found to be superior only in increasing the maternal platelet count, with no difference in maternal and perinatal mortality. In congruence with this meta-analysis, the present study did not determine a statistically significant difference between patients who used dexamethasone in the postpartum period and those who did not with respect to perinatal mortality rates.

In this study, we concluded that HELLP syndrome was a disease that caused perinatal mortality and that it brought about this effect by mainly causing premature deliveries and leading to a low neonatal APGAR score and birth weight. Maternal demographic, clinical, and laboratory data were determined to have no effect on mortality.

The limitation of this study is that it is an observational retrospective study rather than a clinical and prospective study. Our literature review found that studies on this subject only evaluated patients who were diagnosed with HELLP syndrome in the antepartum period. In our study, patients who were diagnosed with HELLP syndrome in both the antepartum and the postpartum period were included. The number of patients included in the present study was higher compared with many other studies. Therefore, we believe that our results will contribute to the literature.

In conclusion, we think that the rate of perinatal mortality can be reduced by identifying patients who are at risk of developing HELLP syndrome and preventing the occurrence of HELLP syndrome or delaying the gestational week of onset. We recommend that patients with HELLP syndrome undergo labor in tertiary hospitals after the required conditions for the mother and the fetus are arranged and an appropriate delivery method is selected. We think that we can decrease the perinatal mortality rate by neonatal examination by experts, and specifically, the provision of the required care based on the 5minute APGAR score.
Acknowledgment: None

Conflict of interest: There is no conflict of interest.

Funding: None.

Availability of data and materials: The data supporting this study is available through the corresponding author upon reasonable request.

Author Contributions: RG, SYT, and TG raised the presented idea. RG, MSI, and SE designed the study. RG conducted the analyses. $R G$ and SYT developed the first draft of the manuscript. All authors contributed to the writing of the paper, and have read and approved the final manuscript.

\section{References}

1. Dusse LM, Alpoim PN, Silva JT, Rios DR, Brandão AH, Cabral AC. Revisiting HELLP syndrome. Clin Chim Acta. 2015;451(Pt B):117-20. Doi: 10.1016/j.cca. 2015. 10.024 .

2. Kınay T, Küçük C, Kayıkçığlu F, Karakaya J. Severe preeclampsia versus HELLP Syndrome: Maternal and perinatal outcomes at $<34$ and $\geq 34$ weeks' gestation. Balkan Med J. 2015;32(4):359-63. Doi: 10.5152/balkanmedj.2015.15777

3. Abildgaard U, Heimdal K. Pathogenesis of the syndrome of hemolysis, elevated liver enzymes, and low platelet count (HELLP): a review. Eur J Obstet Gynecol Reprod Biol. 2013;166(2):117-23. Doi: 10.1016/j.ejogrb. 2012. 09.026 .

4. Rath W, Tsikouras P, Stelzl P. HELLP Syndrome or acute fatty liver of pregnancy: A differential diagnostic challenge: common features and differences. Geburtshilfe Frauenheilkd. 2020;80(5):499-507. Doi: 10.1055/a-10918630 .

5. Sibai BM. Diagnosis, controversies, and management of the syndrome of hemolysis, elevated liver enzymes, and low platelet count. Obstet Gynecol. 2004;103(5 Pt 1):98191. Doi: 10.1097/01.AOG.0000126245.35811.2a.

6. Sibai BM, Spinnato JA, Watson DL, Hill GA, Anderson GD. Pregnancy outcome in 303 cases with severe preeclampsia. Obstet Gynecol. 1984;64(3):319-25. PMID: 6462561.

7. Abramovici D, Friedman SA, Mercer BM, Audibert F, Kao L, Sibai BM. Neonatal outcome in severe preeclampsia at 24 to 36 weeks' gestation: does the HELLP (hemolysis, elevated liver enzymes, and low platelet count) syndrome matter? Am J Obstet Gynecol. 1999;180(1):221-5. Doi: 10.1016/s0002-9378(99)70178-x.

8. ACOG Practice Bulletin No. 202: Gestational Hypertension and Preeclampsia. Obstet Gynecol. 2019;133(1):1. Doi: 10.1097/AOG.0000000000003018.

9. Barfield WD, Committee on fetus and newborn. Standard terminology for fetal, infant, and perinatal deaths. Pediatrics. 2016;137(5):e20160551. Doi: 10.1542/peds. 2016-0551. 
10. American College of Obstetricians and Gynecologists. Hypertension in pregnancy. Report of the American College of Obstetricians and Gynecologists' Task Force on Hypertension in Pregnancy. Obstet Gynecol. 2013;122(5):1122-31. Doi: 10.1097/01.AOG.00004373 82.03963 .88 .

11. Ely DM, Driscoll AK. Infant Mortality in the United States, 2017: Data from the Period Linked Birth/Infant Death File. Natl Vital Stat Rep. 2019;68(10):1-20.

12. Guzel AI, Kuyumcuoglu U, Celik Y. Are maternal and fetal parameters related to perinatal mortality in HELLP syndrome? Arch Gynecol Obstet. 2011;283(6):1227-32. Doi: 10.1007/s00404-010-1534-x.

13. Sadaf N, Haq G, Shukar-ud-Din S. Maternal and foetal outcome in HELLP syndrome at tertiary care hospital. J Pak Med Assoc. 2013;63(12):1500-3. PMID: 24397093

14. Yıldırım G, Güngördük K, Aslan H, Gül A, Bayraktar M, Ceylan Y. Comparison of perinatal and maternal outcomes of severe preeclampsia, eclampsia, and HELLP syndrome. J Turk Ger Gynecol Assoc. 2011;12(2):90-6. Doi: 10.5152/jtgga.2011.22. eCollection 2011.
15. Aydin S, Ersan F, Ark C, Aydın ÇA. Partial HELLP syndrome: Maternal, perinatal, subsequent pregnancy and long-term maternal outcomes. J Obstet Gynaecol Res. 2014;40(4):932-40. Doi: 10.1111/jog.12295.

16. Salmon JE, Heuser C, Triebwasser M, Liszewski MK, Kavanagh D, Roumenina L, et al. Mutations in complement regulatory proteins predispose to preeclampsia: a genetic analysis of the PROMISSE cohort. PLoS Med. 2011;8(3):e1001013. Doi:10.1371/journal.pmed.1001013.

17. van Oostwaard MF, Langenveld J, Schuit E, Papatsonis DN, Brown MA, Byaruhanga RN, et al. Recurrence of hypertensive disorders of pregnancy: an individual patient data metaanalysis. Am J Obstet Gynecol. 2015;212(5): 624.e1-17. Doi: 10.1016/j.ajog.2015.01.009.

18. Sushil C, Ashish M, Raju A. HELLP or Help: A Real Challenge. J Obstet Gynaecol India. 2015;65(3):172-5. Doi: 10.1007/s13224-014-0582-8.

19. Knapen MF, van Altena AM, Peters WH, Merkus HM, Jansen JB, Steegers EA. Liver function following pregnancy complicated by the HELLP syndrome. Br J Obstet Gynaecol. 1998;105(11):1208-10. Doi: 10.1111/j.14710528.1998.tb09977.x. 